\title{
Revisão
}

\section{Long Covid e complicações que impactam na qualidade de vida dos sobreviventes}

\author{
Long Covid and complications that impact the quality of life of survivors
}

\author{
Erlene Roberta Ribeiro dos Santos ${ }^{1}$ (iD Felipe Maia Tardieux² (iD) \\ 1Departamento de Saúde Coletiva do entro Acadêmico da Vitória de Santo Antão - Universidade Federal de Pernambuco, Brasil.
}

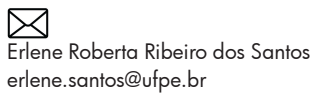

Edited by

Juliana Ramos Andrade

Palavras-chave:

Síndrome pós-aguda

Covid-19

Sars-Cov2

Keywords:

Post-Acute Syndrome

Covid-19

Sars-Cov2

\section{Resumo}

A pandemia de Covid-19 assolou a humanidade desde 2020, tomando proporções nunca antes vivenciadas, atingindo os países de maneira rápida e letal, ceifando vidas em todas as faixas etárias, deixando órfãos, gerando impactos envolvendo diversos aspectos econômicos, sociais, emocionais, epidemiológicos e demais em larga escala, evidenciando a fragilidade dos sistemas de saúde em vários continentes e deixando efeitos que persistem após a infecção para os sobreviventes. Compreendendo essa situação a ser enfrentada pelos sistemas mundiais de saúde escrevemos essas notas com o objetivo de averiguar na literatura as complicações, sintomas e sequelas mais frequentes, que persistem no período de recuperação pela infecção pelo SARS-CoV-2, apresentados pelos sobreviventes. As evidências encontradas revelam o comprometimento de vários sistemas biológicos a partir das complicações e sequelas deixadas pela infecção. Variam desde leves a incapacitantes e impactam diretamente na qualidade de vida dos sobreviventes, assim como, nos custos diretos e indiretos para os sistemas de saúde dos continentes afetados pela pandemia. Compreendendo a magnitude do problema em foco, é necessário recomendar o desenvolvimento de maiores estudos para dimensionar os efeitos da "long Covid" para a sociedade.

\begin{abstract}
The Covid-19 pandemic has ravaged humanity since 2020, taking on proportions never before experienced, reaching countries in a fast and lethal way, claiming lives in all age groups, leaving orphans, generating impacts involving various economic, social, emotional, epidemiological aspects and others on a large scale, highlighting the fragility of health systems on several continents and leaving effects that persist after infection for survivors. Understanding this situation to be faced by the world health systems, we wrote these notes with the aim of investigating in the literature the most frequent complications, symptoms and sequelae, which persist in the recovery period due to SARS-CoV-2 infection, presented by the survivors. The evidence found reveals the involvement of several biological systems from the complications and sequelae left by the infection. They range from mild to disabling and directly impact the quality of life of survivors, as well as the direct and indirect costs for the health systems of the continents affected by the pandemic. Understanding the magnitude of the problem in focus, it is necessary to recommend the development of further studies to measure the effects of "long Covid" for society.
\end{abstract}




\section{Introdução}

A pandemia de Covid-19 desde 2020 impactou a humanidade com proporções nunca antes vistas, avançando de maneira rápida e letal, ceifando vidas em todas as faixas etárias, deixando órfãos, gerando impactos envolvendo diversos aspectos econômicos, sociais, emocionais, epidemiológicos e demais em larga escala, evidenciando a fragilidade dos sistemas de saúde em vários continentes e deixando efeitos que persistem após a infecção para os sobreviventes. ' Compreendendo essa situação a ser enfrentada pelos sistemas mundiais de saúde, escrevemos essas notas com o objetivo de averiguar na literatura as complicações, sintomas e sequelas mais frequentes, que persistem no período de recuperação da infecção pelo SARS-CoV-2, apresentados pelos sobreviventes da Covid-19.

O momento atual é de redução do número de óbitos em muitos países, considerando a vacinação da população e com isso a diminuição da chance de desenvolvimento da forma grave da Covid-19. Entretanto ainda não é sabido o tempo exato de duração do efeito de proteção da vacina, quando observados os casos de pessoas imunizadas que já desenvolveram novamente a doença e foram a óbito. Além disso, as descobertas para o tratamento ainda são esperadas, o que reforça a necessidade dos governantes se dedicarem à discussão da importância dos efeitos gerados pela pandemia para os sobreviventes, que variam de condições incapacitantes temporárias a permanentes, só o tempo nos permitirá avaliar as consequências dessa situação. ${ }^{2}$

A necessidade de identificar e gerenciar novas situações para o cuidado na assistência à saúde está sendo investigada a partir de novos estudos que utilizam o termo "Long Covid", cuja definição se edifica como detecção ou persistência de sintomas agudos pós infecção oriunda da Covid-19, sintomas residuais que permanecem após a recuperação pela infecção aguda, ou novos sintomas que aparecem nos assintomáticos, ou sintomatologia leve com testagem positiva. O diagnóstico de "Long Covid" também pode ser definido como a persistência ou surgimento dos sintomas, não associados a nenhuma outra causa específica, após dias de recuperação pela infecção por SARS-CoV-2., ${ }^{3,4}$

Dentre os determinantes causais de "Long Covid", podem ser destacados fatores tais como: Longo período de permanência em internamento mediante o auxílio de aparelhos para manutenção da vida; sobrecarga de medicamentos para o organismo durante a internaçã $0^{5}$; exaustão do sistema imunológico; alto nível de estresse e ansiedade por medo da morte; Longo tempo de reabilitação; receio de exposição a novo processo de contaminação, dentre outras que envolvem aspectos econômicos e sociais advindos da condição incapacitante, a depender da sequela a ser tratada. ${ }^{6}$

Em Wuhan, China, um estudo de coorte expôs que seis meses após a infecção aguda, infectados pela Covid-19 indicaram sequelas como fadiga, fraqueza muscular, dificuldades para dormir, ansiedade ou depressão. ${ }^{7}$ Ainda nesta linha, pesquisadores realizaram um estudo observacional retrospectivo em hospitais da Espanha, que evidenciou a seriedade das sequelas nos sobreviventes à hospitalização devido a Covid-19. O estudo espanhol apontou que $63,9 \%$ dos sobreviventes relataram alguma sequela passados seis meses da alta hospitalar e $20 \%$ voltaram ao serviço de emergência. ${ }^{8}$

Desse modo, a "Long Covid" se revela como uma condição multissistêmica vinculada a um conjunto de complicações dos vários sistemas biológicos isolados ou combinados, destacando-se as sequelas respiratórias, cerebrovasculares a exemplo do aumento dos casos de acidente vascular encefálico (AVE) e alterações neurológicas, cardiovasculares, desordem mental, esgotamento físico, fadiga intensa incapacitante para tarefas ordinárias, cefaleias e demais mialgias. ${ }^{5} \mathrm{O}$ sistema endócrino também é afetado pela permanência das sequelas durante a recuperação dos sobreviventes ${ }^{9}$, conforme podemos observar no quadro 1 que associa os sistemas biológicos aos sintomas e complicações mais frequentes para os sobreviventes.

Quadro 1. Sistemas biológicos, áreas afetadas e complicações persistentes pós Covid-19.

\begin{tabular}{|c|c|c|}
\hline Estudos & Áreas afetadas & Complicaçōes mais frequentes \\
\hline $\begin{array}{l}\text { Dennis et al. }{ }^{10} \\
\text { Lui et al.9 } \\
\text { Orrù et al. }{ }^{11} \\
\text { Touyz et al. }{ }^{12}\end{array}$ & Cardiovascular & $\begin{array}{l}\text { Hipertensão arterial, infarto agudo } \\
\text { do miocárdio (IAM), palpitaçôes, } \\
\text { miocardite. }\end{array}$ \\
\hline $\begin{array}{l}\text { Lui et al. } \\
\text { Orrù et al." }\end{array}$ & Endócrina & Disfunções na tireoide, diabetes. \\
\hline $\begin{array}{l}\text { Lui et al. } \\
\text { Orrù et al. }{ }^{11}\end{array}$ & Respiratória & Dispneia, tosse, anosmia. \\
\hline $\begin{array}{l}\text { Chippa, Aleem e Anjum }{ }^{13} \\
\text { Dennis et al.10 } \\
\text { Huang et al. } \\
\text { Orrù et al. } .^{71} \\
\text { Touyz et al. }{ }^{12} \\
\text { Valença, Andrade e Peres }{ }^{5}\end{array}$ & Locomotora & $\begin{array}{l}\text { Esgotamento físico, dispneia e } \\
\text { fadiga. }\end{array}$ \\
\hline $\begin{array}{l}\text { Chippa, Aleem e Anjum } \\
\left(20211^{13}\right. \\
\text { Huang et al. } \\
\text { Orrü et al.1 } \\
\text { Valença, Andrade e Peres } \\
(2020)^{5}\end{array}$ & $\begin{array}{l}\text { Músculo- } \\
\text { esquelética }\end{array}$ & $\begin{array}{l}\text { Dor nas articulações e dor ou } \\
\text { fraqueza muscular. }\end{array}$ \\
\hline Orrù et al."1 & Digestiva & $\begin{array}{l}\text { Dor abdominal, náusea, diarreia, } \\
\text { anorexia e diminuição do apetite. }\end{array}$ \\
\hline Dennis et al. ${ }^{10}$ & Renal & Comprometimento leve dos rins. \\
\hline $\begin{array}{l}\text { Chippa, Aleem e Anjum } 13 \\
\text { Dennis et al.10 } \\
\text { Lui et al. } \\
\text { Orù et al.111 } \\
\text { Touyz et al.12 } \\
\text { Valença, Andrade e Peres }\end{array}$ & Neurológica & $\begin{array}{l}\text { Acidente vascular encefálico, } \\
\text { encefalopatia, meningoencefalite, } \\
\text { convulsōes, comprometimento } \\
\text { cognitivo, cefaleia, distúrbios } \\
\text { do sono, tontura, delírio, } \\
\text { disautonomia. }\end{array}$ \\
\hline $\begin{array}{l}\text { Chippa, Aleem e Anjum }{ }^{13} \\
\text { Huang et all? } \\
\text { Orrù et al. }{ }^{7}\end{array}$ & $\begin{array}{l}\text { De natureza } \\
\text { emocional }\end{array}$ & $\begin{array}{l}\text { Ansiedade, crises de pânico, } \\
\text { depressão, distúrbios do sono. }\end{array}$ \\
\hline
\end{tabular}

Nota: Baseado na caracterização dos sistemas de acordo com Taboada e colaboradores. ${ }^{14}$ Instituto Nacional de Excelência em Saúde e Cuidados (NICE). 
Quando os sintomas persistem além de 3 ou 12 semanas, os resultados das condições patológicas foram ordenados em duas categorias, sendo a primeira "Long Covid pós-agudo" e "Covid crônico". Nas duas situações as condições são caracterizadas por uma síndrome do sistema que requer uma ação multidisciplinar integrada capaz de tratar os sintomas físicos residuais assim como os desconfortos sociais e emocionais."

Faz-se necessário destacar que os agravos que acometem o sistema renal, a exemplo da lesão renal aguda, frequentemente levam ao óbito, assim não progredindo ao estágio de long Covid.15

Evidências de estudo prévio realizado na ltália, enfatizam a prevalência de sintomas físicos, neurológicos, psicológicos e fisiológicos, que impactam na qualidade de vida dos sobreviventes. Foi realizada aplicação de escala online com 507 participantes, em sua maioria predominou o sexo feminino 416/507, cujos principais sintomas de "Long Covid" foram cefaleia, fadiga, mialgia, dores articulares, deficiência cognitiva, perda de concentração e perda do olfato. Associadas a essas condições, a maioria dos respondentes apresentaram níveis de significância estatística para insônia $(p<0,05)$, assim como um déficit geral da qualidade de vida."

Em outro estudo é possível observar comprometimento no sistema endócrino, que mostra maior vulnerabilidade para a população feminina quando observadas as disfunções na tireoide e no sistema autoimune durante o período de recuperação da infecção. ${ }^{9}$ Além disso, em uma pesquisa prospectiva realizada no Reino Unido, estudiosos concluíram que em 70\% dos indivíduos com baixo risco de mortalidade por Covid-19, apresentaram sintomas contínuos durante 16 semanas, e revelaram comprometimento em um ou mais órgãos. ${ }^{10}$

Por isso é importante estarmos em alerta para a gravidade da situação de sobreviventes assintomáticos, que de maneira silenciosa apresentam alterações significativas no funcionamento dos sistemas biológicos, podendo apresentar consequências danosas para o funcionamento hematopoiético, imunidade, função renal, condições hepáticas, neurológicas, cardiológicas e respiratórias considerando que todos os órgãos podem ser afetados durante a infecção. ${ }^{5}$

Os sistemas de saúde além do enfrentamento para atender às demandas da "Long Covid" para os sobreviventes, necessita priorizar o planejamento de redes de apoio para os profissionais, que já estão nos serviços de assistência intensiva, combatendo a pandemia dede o início. Estes estão exacerbadamente esgotados, também tiveram a sua qualidade de vida afetada, em razão da empatia com as famílias enlutadas dos pacientes, pessoas próximas e colegas de trabalho que morreram ou estão enfrentando complicações por "Long Covid", além do estresse pós-traumático que vivenciam no cotidiano das unidades de saúde. ${ }^{16}$
Essa nova situação é preocupante e precisa ser incorporada às políticas de saúde, pois implicará em diagnóstico precoce e aparelhamento dos sistemas para intervenção nas terapias de reabilitação dos sobreviventes, paralelamente as ações de imunização, uso de máscaras, distanciamento social, cuidados para evitar o contágio e manutenção dos atendimentos aos infectados, que ainda se dá no âmbito das unidades de assistência médica.

Com base nessas notas é importante destacar que os preditores da "Long Covid" ainda necessitam de vastos estudos no acompanhamento, organização e planejamento das políiticas de saúde, pois o enfrentamento dessa nova situação epidemiológica, emerge em momento de curso da pandemia, o que gerará custos diretos e indiretos para a sociedade por tempo indeterminado.

Erlene Roberta Ribeiro dos Santos

https://orcid.org/0000-0003-3334- 3408

Felipe Maia Tardieux

https://orcid.org/0000-0001-8275-1243

\section{Referências}

1. WHO Coronavirus (COVID-19) Dashboard. [Internet]. World Health Organization; 2021 Available from: https:// covid 19. who.int.

2. Hopkins C, Burges Watson DL, Kelly C, Leary V and Smith $B C$. Managing long covid: don't overlook olfactory dysfunction. Bmi 2020;370:m3736 Doi:10.1136/bmj. m3736

3. Marshall $M$. The lasting misery of coronavirus long-haulers. Nature 2020;585(7825):339-341 Doi: 10.1038/ d41586-020-02598-6

4. Mendelson M, Nel J, Blumberg L, Madhi SA, Dryden M, Stevens $W$ and Venter FWD. Long-COVID: An evolving problem with an extensive impact. S Afr Med J 2020;1 11 (1): 10-12 Doi: 10.7196/samj.2020.v1 11 i1 1.15433

5. Valença MM, Andrade JRd and Peres MFP. Long Covid and persistent headache. Headache Medicine 2020;1 1(4):7980 Doi: 10.48208/HeadacheMed.2020.23

6. Long COVID: let patients help define long-lasting COVID symptoms. Nature 2020;586(7828): 170 Doi: 10.1038/ d41586-020-02796-2

7. Huang $C$, Huang L, Wang Y, Li X, Ren L, Gu X, .. Cao B. 6-month consequences of COVID-19 in patients discharged from hospital: a cohort study. Lancet 2021;397(10270):220232 Doi: 10.1016/s0140-6736(20)32656-8

8. Romero-Duarte Á, Rivera-lzquierdo M, Guerrero-Fernández de Alba I, Pérez-Contreras M, Fernández-Martínez NF, Ruiz-Montero R, . . Cárdenas-Cruz A. Sequelae, persistent symptomatology and outcomes after COVID-19 hospitalization: the ANCOHVID multicentre 6-month follow-up study. BMC Med 2021;19(1):129 Doi: 10.1186/ s12916-021-02003-7 
9. Lui DTW, Lee $\mathrm{CH}$, Chow WS, Lee ACH, Tam AR, Pang P, . . . Lam KSL. Long COVID in Patients with Mild to Moderate Disease: Do Thyroid Function and Autoimmunity Play a Role? Endocr Pract 2021;Doi: 10.1016/i.eprac.2021.06.016

10. Dennis A, Wamil M, Alberts J, Oben J, Cuthbertson DJ, Wootton $D, \ldots$ Banerjee A. Multiorgan impairment in low-risk individuals with post-COVID-19 syndrome: a prospective, community-based study. BMJ Open 202 1; 1 1 (3):e048391 Doi: 10.1136/bmjopen-2020-048391

11. Orrù G, Bertelloni D, Diolaiuti F, Mucci F, Di Giu seppe $M$, Biella $M$, . . . Conversano C. Long-COVID Syndrome? A Study on the Persistence of Neurological, Psychological and Physiological Symptoms. $2021 ; 9(5): 575$ Doi: 10.3390/healthcare9050575

12. Touyz RM, Boyd MO, Guzik T, Padmanabhan S, McCallum L, Delles C, . . . Berry C. Cardiovascular and renal risk factors and complications associated with COVID-19. CJC Open 2021;Doi: 10.1016/i.cjco.2021.05.020
13. Chippa V, Aleem A and Anjum F Post Acute Coronavirus (COVID-19) Syndrome. StatPearls. Treasure Island (FL): StatPearls Publishing Copyright (C) 2021, StatPearls Publishing LLC.; 2021.

14. Taboada M, Moreno E, Cariñena A, Rey T, Pita-Romero R, Leal $S, \ldots$ Seoane-Pillado T. Quality of life, functional status, and persistent symptoms after intensive care of COVID-19 patients. Br J Anaesth 2021;126(3):e 110 -e 113 Doi: 10.1016/i.bja.2020.12.007

15. Higgins V, Sohaei D, Diamandis EP and Prassas I. COVID-19: from an acute to chronic disease? Potential long-term health consequences. Crit Rev Clin Lab Sci 2021;58(5):297. 310 Doi: 10.1080/10408363.2020.1860895

16. Orrù G, Marzetti F, Conversano C, Vagheggini G, Miccoli M, Ciacchini R, . . . Gemignani A. Secondary Traumatic Stress and Burnout in Healthcare Workers during COVID-19 Outbreak. Int J Environ Res Public Health 2021;18(1):337 Doi: 10.3390/ijerph 18010337 